\title{
Targeting epithelium-expressed sialyl Lewis glycans improves colonic mucosal wound healing and protects against colitis
}

\author{
Matthias Kelm, ${ }^{1}$ Miguel Quiros, ${ }^{1}$ Veronica Azcutia, ${ }^{1}$ Kevin Boerner, ${ }^{1}$ Richard D. Cummings, ${ }^{2}$ \\ Asma Nusrat, ${ }^{1}$ Jennifer C. Brazil,, ${ }^{1}$ and Charles A. Parkos ${ }^{1}$ \\ 'Department of Pathology, University of Michigan, Ann Arbor, Michigan, USA. ${ }^{2}$ Department of Surgery, Beth Israel \\ Deaconess Medical Center, Harvard Medical School, Boston, Massachusetts, USA.
}

\begin{abstract}
Dysregulated healing of injured mucosa is a hallmark of many pathological conditions, including inflammatory bowel disease. Mucosal injury and chronic intestinal inflammation are also associated with alterations in epithelial glycosylation. Previous studies have revealed that inflammation-induced glycan sialyl Lewis A on epithelial CD44v6 acts as a ligand for transmigrating PMNs. Here we report that robust sialylated Lewis glycan expression was induced in colonic mucosa from individuals with ulcerative colitis and Crohn disease as well as in the colonic epithelium of mice with colitis induced by dextran sodium sulfate (DSS). Targeting of sialylated epithelial Lewis glycans with mAb GM35 reduced disease activity and improved mucosal integrity during DSSinduced colitis in mice. Wound healing studies revealed increased epithelial proliferation and migration responses as well as improved mucosal repair after ligation of epithelial sialyl Lewis glycans. Finally, we showed that CM35-mediated increases in epithelial proliferation and migration were mediated through activation of kinases that signal downstream of CD44v6 (Src, FAK, Akt). These findings suggest that sialylated Lewis glycans on CD44v6 represent epithelial targets for improved recovery of intestinal barrier function and restitution of mucosal homeostasis after inflammation or injury.
\end{abstract}

Authorship note: JCB and CAP contributed equally to this work.

Conflict of interest: The authors have declared that no conflict of interest exists.

Copyright: (c) 2020, American Society for Clinical Investigation.

Submitted: December 23, 2019

Accepted: May 13, 2020

Published: June 18, 2020

Reference information: JCI Insight. 2020;5(12):e135843.

https://doi.org/10.1172/jci.

insight.135843.

\section{Introduction}

The intestinal epithelium forms a critical barrier separating luminal antigens from underlying mucosal tissues. Epithelial wounds in the intestine resulting from mechanical damage or inflammation must be efficiently repaired in order to maintain gut homeostasis and ensure adequate absorption of nutrients. Mucosal wound closure in the gut is mediated by a complex spatiotemporal interplay between intestinal epithelial cells (IECs) and innate and adaptive immune cells that are recruited into damaged tissues. Reepithelization of denuded intestinal mucosa is facilitated by migration of epithelial cells from wound-adjacent crypts over the wound bed, accompanied by epithelial proliferation and differentiation to reestablish tissue architecture and barrier function (1-3).

Epithelial cell migration is a complicated process that is, in part, facilitated by binding interactions between migrating cells and the underlying extracellular matrix. The motility of epithelial cells also requires activation of intracellular signaling cascades triggered by engagement of cell surface receptors. The CD44 family of cell adhesion molecules plays a well-described role in epithelial cell-cell and cell-matrix adhesive interactions (4). In particular, the heavily glycosylated cancer-associated CD44 family member CD44v6 is known to play an important role in epithelial migration and proliferation (5). In previous studies investigating mechanisms of intestinal inflammation and repair, we produced an anti-sialyl Lewis glycan mAb (GM35) that specifically recognized O-glycan-linked sialylated Lewis glycans expressed on CD44v6 in inflamed human intestinal mucosa. Further, in addition to marking inflamed colonic mucosa, GM35 targeting of epithelial sialyl Lewis A (sLe ${ }^{a}$ /CD44v6 blocked transepithelial migration of human neutrophils. Importantly, these studies revealed that, in addition to binding the human glycan sLe ${ }^{\mathrm{a}}$, GM35 also recognized an additional Lewis glycan family member, sialyl Lewis $\mathrm{C}\left(\mathrm{sLe}^{\mathrm{c}}\right)$, which serves as a precursor to sLe $\mathrm{e}^{\mathrm{a}}$ and is expressed by both humans and mice. Furthermore, targeting of sialyl Lewis glycans on CD44v6 with GM35 in vivo blocked PMN transmigration to the small intestine and improved epithelial barrier function $(6,7)$. 
The importance of glycan-mediated binding events for restoration of mucosal homeostasis after injury is supported by studies on galectin 2 and galectin 4, which have been shown to promote reepithelization of intestinal wounds by enhancing binding interactions between epithelial cells and $\beta$-galactoside-containing glycans on cell surface and matrix glycoproteins (8). Similarly, other studies have demonstrated a role for cell surface N-glycans in organizing cell-cell junction dynamics and promoting epithelial cell motility (9). However, despite previous reports of glycan-regulated epithelial mobility and intestinal wound repair, the potential of epithelial sialyl Lewis glycans as targets for amelioration of intestinal inflammation and promotion of mucosal wound healing have not been explored to date.

In this study, we demonstrated that antibody-mediated targeting of sialyl Lewis glycan epitopes on epithelial CD44v6 protected against colitis induced by dextran sodium sulfate (DSS) and promoted repair of damaged intestinal mucosa in vivo. Our data demonstrated that the protective or prorepair effects of GM35 were not mediated by reduced PMN mucosal trafficking, but rather were a direct consequence of increased epithelial migration and proliferation responses triggered by sialylated Lewis glycan engagement. Because delayed wound repair is a pathological hallmark of multiple conditions, including inflammatory bowel disease (IBD) and anastomotic failure, targeting inflammation-induced carbohydrate signatures, including sialyl Lewis glycans, to reduce inflammation and restore gut homeostasis may represent a promising new therapeutic opportunity.

\section{Results}

Increased expression and colocalization of sialyl Lewis glycans and CD44v6 in inflamed colonic mucosa. Previous studies have shown that the anti-glycan mAb GM35 binds to sLe $\mathrm{e}^{\mathrm{a}}$ on CD44v6 expressed by inflamed epithelium and reduces PMN transepithelital migration (TEpM) $(6,7)$. However, the potential of sialylated Lewis glycans as targets to reduce intestinal inflammation in vivo has not been explored to date. Immunohistochemical analyses of normal intestinal mucosa (Figure 1A) or noninvolved/noninflamed intestinal mucosa from patients with Crohn disease (Figure 1C) revealed minimal basal expression of sialylated Lewis glycans. In contrast, significantly increased expression of sialylated Lewis glycans was detected by mAb GM35 in inflamed intestinal mucosa from patients with ulcerative colitis and Crohn disease (Figure $1, \mathrm{~B}, \mathrm{D}$, and $\left.\mathrm{E},{ }^{* *} P<0.01\right)$. Immunofluorescence analyses were performed to determine expression and localization of sialyl Lewis glycans/CD44v6 in murine colonic mucosa. In normal colonic epithelium, expression of sialyl Lewis glycans (shown in green) and CD44v6 (shown in red) were detected in crypt bases, consistent with previous reports identifying CD44v6 as a marker of crypt cells in normal murine intestinal mucosa (10). Low levels of expression of sialyl Lewis glycans/CD44v6 were also detected at the very apical or luminal aspect of the colonic intestinal epithelium (white arrows, Figure 1F). Colocalization of sialyl Lewis glycans and CD44v6 was also observed (merged images, Figure 1F). Importantly, in inflamed epithelium, detection of sialyl Lewis glycans and CD44v6 was increased, with expression observed along the entire length of colonic crypts. Furthermore, consistent with specific recognition of glycans on CD44v6 by GM35, colocalization of CD44v6 and sialyl Lewis glycans was observed along the length of inflamed colonic epithelial crypts in vivo. In contrast, there was no colocalization of sialyl Lewis glycans recognized by GM35 with the highly O-glycosylated mucin protein and goblet cell marker Muc-2 in normal or inflamed intestinal mucosa (Figure 1G). Furthermore, Muc-2 staining revealed the expected loss of goblet cells after DSS-induced colitis (11).

Targeting sialyl Lewis glycans on CD44v6 protects against DSS-induced colitis. Given the observed inflammation-induced increases in sialyl Lewis glycans on epithelial CD44v6, experiments were performed to probe the functional effect(s) of targeting these glyco-epitopes in vivo. Disease progression in DSS-induced colitis was assessed in mice treated with GM35 to ligate sialylated Lewis glycans. Systemic treatment with mAb GM35 (to target sialyl Lewis glycans on CD44v6) significantly decreased disease severity relative to mice treated with isotype control IgG mAb on days 5 to 8 in the DSS model of acute colitis $\left({ }^{* *} P<\right.$ $0.01,{ }^{* *} P<0.001$, Figure $\left.2 \mathrm{~A}\right)$. Analysis of body weight revealed that GM35 treatment before and during $2.5 \%$ DSS administration significantly reduced weight loss compared with control IgG-treated animals $\left({ }^{*} P<0.05\right.$, Figure $2 \mathrm{C}$ ). In addition, histological analyses of colonic sections from mice treated with GM35 revealed that lower disease activity and weight loss scores correlated with a significant reduction in mucosal injury ( ${ }^{*} P<0.05$, Figure 2, D and E). Importantly, GM35-treated mice showed less histological evidence of injury, highlighted by preservation of surface epithelium and significantly less mucosal ulceration. In contrast, animals treated with isotype control mAb plus 2.5\% DSS had large areas of ulceration 
A

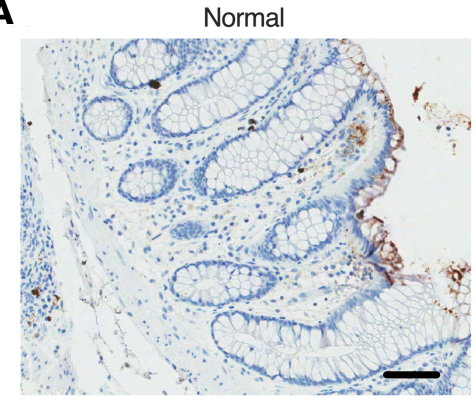

C

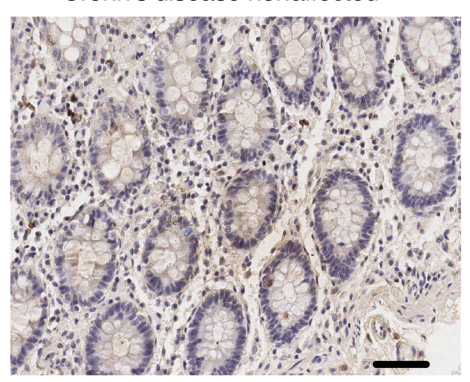

B

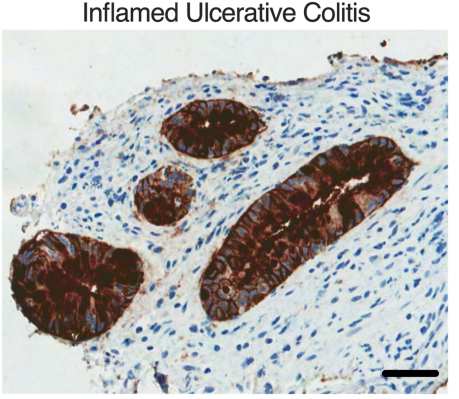

D

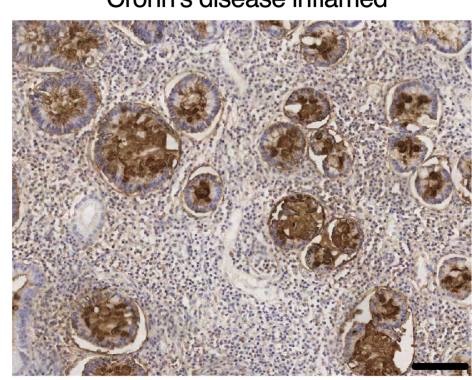

E
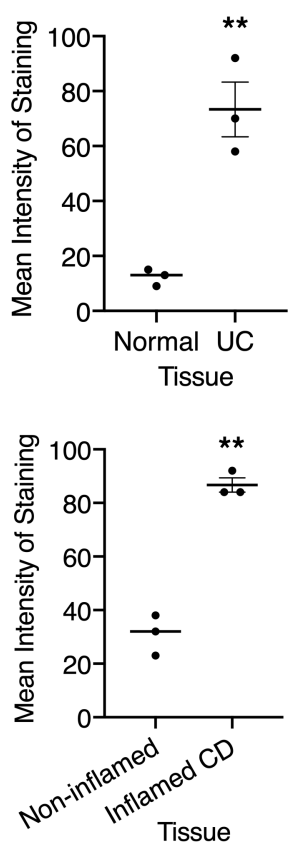

$\mathbf{F}$
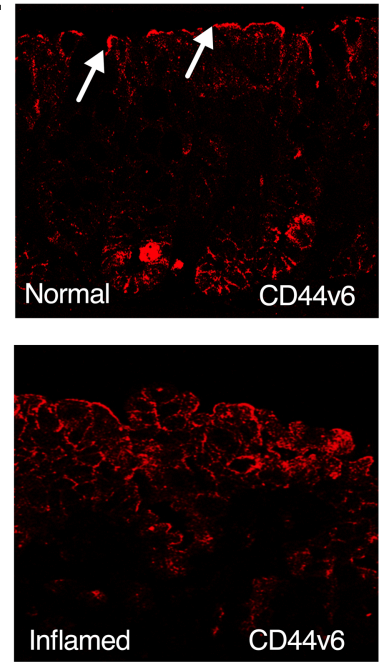

G
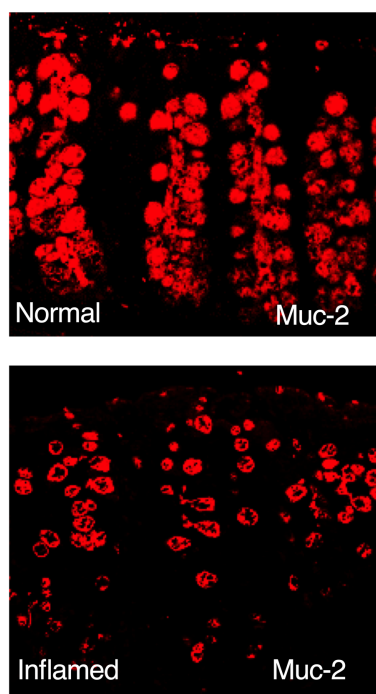
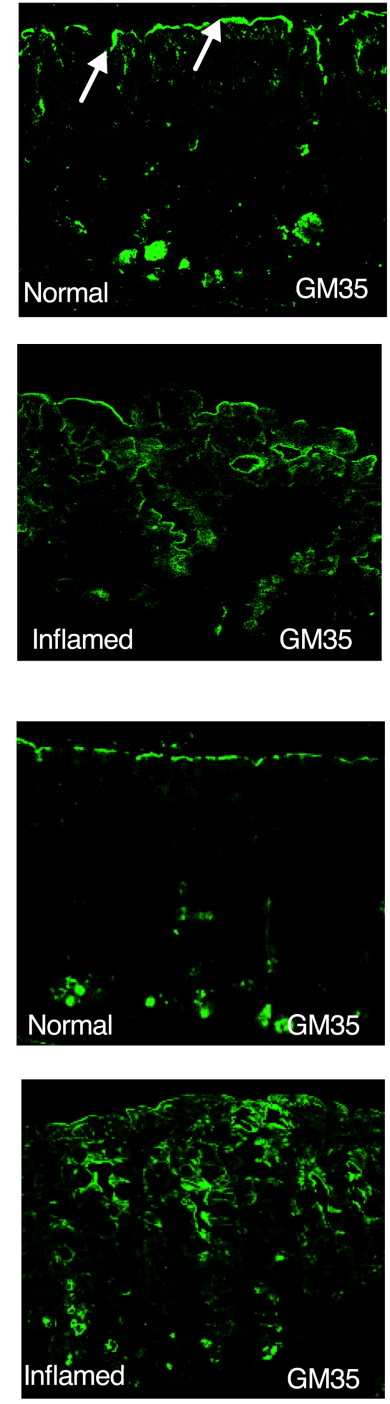
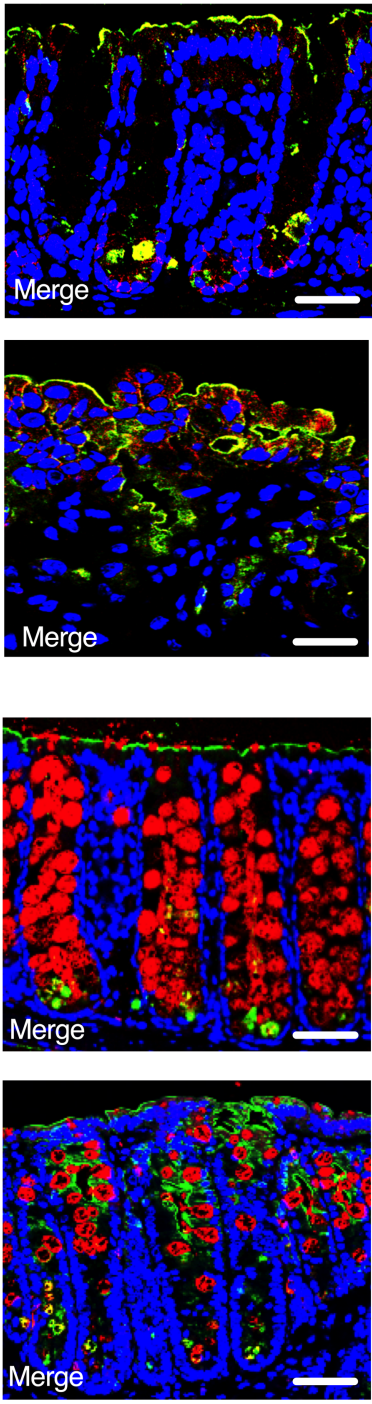

Figure 1. Increased expression of CD44v6 and sialyl Lewis glycans in inflamed murine and human intestinal mucosa. Immunohistochemical analysis of healthy colonic mucosa and colonic mucosa from patients with IBD. Representative images from normal uninflamed mucosa (A) are compared with active ulcerative colitis (UC) (B). Noninflamed mucosa (C) and adjacent inflamed tissue (D) from Crohn disease (CD) are also compared. (E) Quantification of intensity of staining from $n=3$ normal colonic mucosa or colonic mucosa from $n=3$ patients with UC or CD. Data are shown as means \pm SEM and were analyzed by 1-way ANOVA followed by Tukey's post hoc testing. ${ }^{*} P<0.01$. Expression of CD44v6 (red), Muc-2 (red), or sialyl Lewis glycans stained with GM35 (green) in representative images of normal colonic mucosa (F) and inflamed colonic mucosa (C) of WT [57BL/6] mice given $2.5 \%$ DSS for 5 days. Images are representative of $n=3$ independent experiments. Scale bar: $10 \mu \mathrm{m}$. 
together with increased numbers of infiltrated immune cells (Figure 2D, black arrows). Analysis of PMN migration into the proximal colon in response to a solution of luminally applied LTB4 revealed no difference in transmigrated PMNs mediated by GM35 (Figure 2, F and G), suggesting that protective effects observed during DSS-induced colitis are not a result of reduced PMN trafficking.

To determine whether GM35 targeting of epithelial sialyl Lewis glycans was protective against colitis without $\mathrm{mAb}$ pretreatment, experiments were performed in which mice were exposed to DSS followed by treatment with GM35 on days 1, 3, 6, and 8. As was observed above and as shown in Figure 2, GM35 targeting of sialylated epithelial Lewis glycans significantly reduced DSS-induced weight loss and mucosal injury compared with IgG- or PBS-treated control mice $\left({ }^{*} P<0.05 * * P<0.01\right.$, Supplemental Figure 1; supplemental material available online with this article; https://doi.org/10.1172/jci.insight.135843DS1). Taken together, these findings suggest that epithelial sialyl Lewis glycans on CD44v6 are upregulated during inflammation in vivo and can be selectively targeted to reduce disease severity during colitis.

Targeting sialyl Lewis glycan epitopes on CD44v6 improves healing of biopsy-induced mucosal wounds in vivo. We next examined the direct effects of targeting sialyl Lewis glycans with mAb GM35 on colonic wound healing in vivo (schematic, Figure 3A) using a well-characterized biopsy wounding model (12). As can be seen in Figure 3B, direct injection of GM35 in wound beds 24 hours after biopsy resulted in significantly increased rates of wound closure 1 day later (day 2) compared with injection with an IgG control $\mathrm{mAb}(34 \% \pm 1.2 \%$ vs. $\left.25 \% \pm 1.7 \%,{ }^{*} P<0.01\right)$. A similar statistically significant increase in wound healing downstream of wound bed injection of GM35 was also observed 72 hours after biopsy/day 3 ( $46 \% \pm 3.7 \%$ vs. 36\% $\pm 1.9 \%$, $* * * * P<0.0001$ ). Similarly, systemic treatment (i.p. injection of GM35) resulted in significantly increased colonic mucosal wound healing relative to injection of an IgG control mAb on day $2(42 \% \pm 2.7 \%$ vs. $28 \%$ $\left.\pm 1.7 \%,{ }^{* * * *} P<0.0001\right)$ and day $3(50 \% \pm 3.3 \%$ vs. $38 \% \pm 2.2 \%, * * * * P<0.0001)$ after injury (Figure $3, \mathrm{C}$ and D). These data demonstrate that sialyl Lewis glycans can be selectively targeted in vivo to significantly enhance mucosal wound closure. Furthermore, these findings suggest that inflammation-induced intestinal glycans represent potential targets to improve mucosal repair in chronic conditions such as IBD.

Given that previous in vitro studies have shown inhibition of PMN migration across monolayers of human IECs downstream of targeting sLe $\mathrm{e}^{\mathrm{a}}$ on CD44v6 (7), we examined the effect(s) of GM35 treatment on immune cell infiltration of mucosal wounds in vivo. Consistent with the lack of inhibition of PMN trafficking to the colon mediated by GM35 (Figure 2, G and F), flow cytometric analyses of colonic wound-infiltrating myeloid cells revealed no significant differences in recruitment of neutrophils, monocytes, macrophages, or eosinophils to wounds after treatment with GM35 compared with an IgG control mAb (Supplemental Figure 2). Importantly, these findings highlight that GM35-mediated improvement in colonic mucosal wound healing was a result of epithelial rather than immune cell-mediated effects. Indeed, no expression of sLe ${ }^{\mathrm{a}} / \mathrm{sLe}^{\mathrm{c}}$ on neutrophils, monocytes, B cells, T cells, basophils, or eosinophils was observed (Supplemental Figure 3), demonstrating restricted epithelial expression of sialylated Lewis glycans on CD44v6 recognized by GM35 in vivo. In addition to improved mucosal repair, labeling of wounds with GM35 and anti-CD44v6 mAbs revealed increased expression of sialyl Lewis glycans/CD44v6 along the entire length of epithelial crypts directly adjacent to wound beds (Figure 3E), consistent with the induction of expression of sialylated Lewis glycans observed during DSS-induced colitis (Figure 1B). Furthermore, epithelial colocalization of sialyl Lewis glycans and CD44v6 was observed. Taken together, these results highlight the increased nature of expression of sialylated Lewis glycans on epithelial CD44v6 after wounding or inflammation in the colon.

Given that coordinated epithelial cell proliferation and migration are necessary for mucosal wound healing, we examined the effects of targeting sialyl Lewis glycans on IEC proliferation, focusing analyses on crypts adjacent to biopsy-induced mucosal wounds. As can be seen in Figure 3F, i.p. injection of GM35 resulted in a significant increase in the number of proliferating epithelial cells in wound-adjacent crypts compared with IgG-injected controls $(66 \% \pm 5.3 \%$ vs. $51 \% \pm 7.02 \% * * * P>0.001)$. These results suggest that increased epithelial migration and proliferation contribute to faster wound repair downstream of sialyl Lewis glycan targeting in vivo.

Targeting sialyl Lewis glycan epitopes on CD44v6 by GM35 increases migration and proliferation of human IECs. Given the increased colonic wound healing observed downstream of targeting of sialyl Lewis glycans in vivo, we performed experiments directed at analysis of wound closure using monolayers of human epithelial cells. IEC monolayers were treated with GM35, a second commercially available anti-sLe ${ }^{a}$ mAb NS19-9, an anti-peptide CD44v6 mAb, or an IgG control mAb. As can be seen in Figure 4A, treatment of T84 IECs with GM35 or NS19-9 resulted in significantly enhanced wound 
A

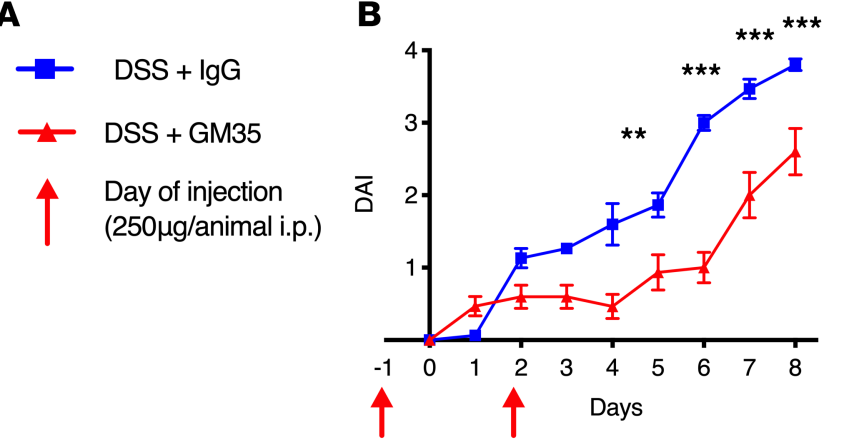

C

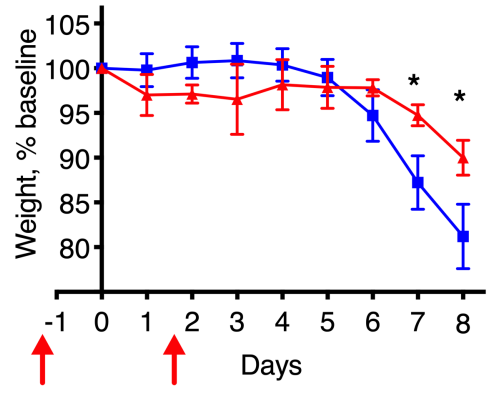

D

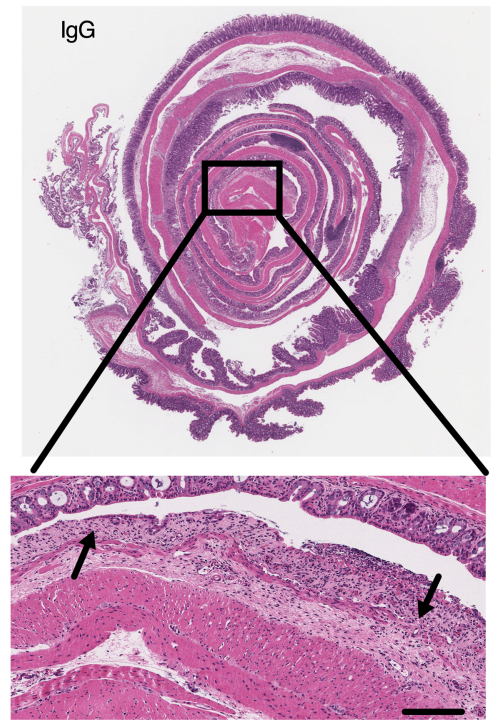

GM35

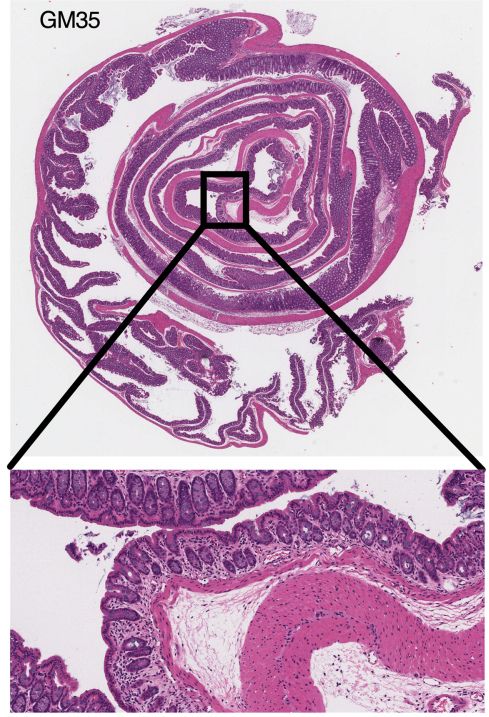

Proximal Colon

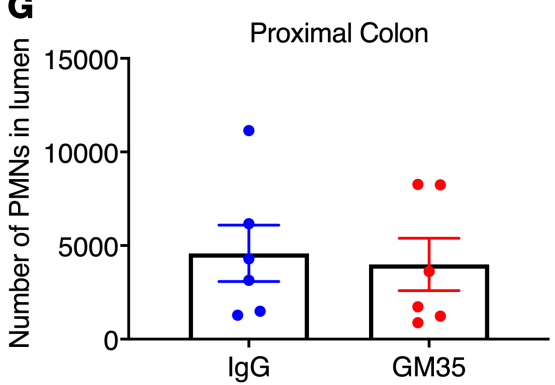

E

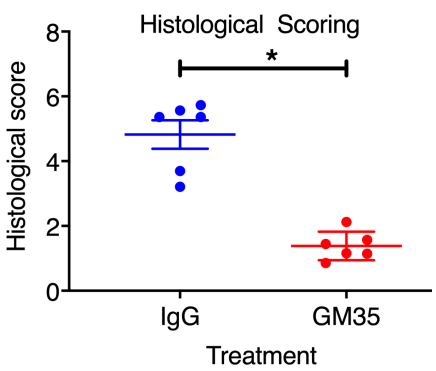

Figure 2. Targeting sialyl Lewis glycan epitopes on CD44v6 with GM35 protects against acute DSS-induced colitis in vivo. (A and B) Disease Activity Index consisting of body weight changes, stool consistency, and presence of occult blood or (C) weight change alone in WT C57BL/6) mice i.p. injected with $250 \mu \mathrm{g}$ mAb GM35 or control $\lg \mathrm{1} 1$ day before and 2 days after administration of water containing $2.5 \%$ DSS (6 mice per group). Mice were euthanized and tissues harvested at day 8. Data are shown as means $\pm \mathrm{SEM},{ }^{* *} P<$ 0.01 , ${ }^{* *} P<0.001$ ( $n=3$ independent experiments). (D) Representative histological images of Swiss rolls of whole colons with magnifications of the rectum to assess levels of DSS-induced colitis. Left image from mouse treated with IgG, right image mouse treated with mAb GM35. Scale bar: $50 \mu \mathrm{m}$. (E) Histological scoring of colonic tissue after 8 days of DSS treatment. Histology was scored by 2 independent investigators. Data are shown as means \pm SEM and were analyzed by 1-way ANOVA followed by Tukey's post hoc testing. ${ }^{*} P<0.05$. (F) Proximal colon loop. (C) Number of PMNs recruited to the lumen of the proximal colon in response to $1 \mathrm{nM}$ LTB4 after luminal injection of GM35 (6 mice) or an isotype control mAb (6 mice).

closure 12, 18, and 24 hours after wounding when compared with control IgG-treated or anti-CD44v6 $\mathrm{mAb}$-treated epithelial cells $\left({ }^{*} P<0.05,{ }^{* *} P<0.01\right)$. In addition, staining of T84 IEC monolayers 24 hours after scratch wounding revealed expression and colocalization of GM35 binding sialylated Lewis glycans and CD44v6 at the wound edge (Figure 4B). Similar to effects observed in T84 IECs, increased epithelial migration/wound closure was also observed in 2-dimensional (2D) monolayers of primary human colonoid-derived epithelial cells (Figure 4C) after targeting sialyl Lewis glycans with GM35 $\left(77.2 \% \pm 3.4 \%\right.$ vs. $\left.59.8 \% \pm 7.0 \%,{ }^{* *} P<0.01\right)$.

Given the observations of sialyl Lewis glycan-mediated increases in epithelial proliferation in vivo (Figure $3 \mathrm{~F}$ ), we examined epithelial proliferation rates 18 hours after wounding of T84 IECs (Figure 4, D 
A

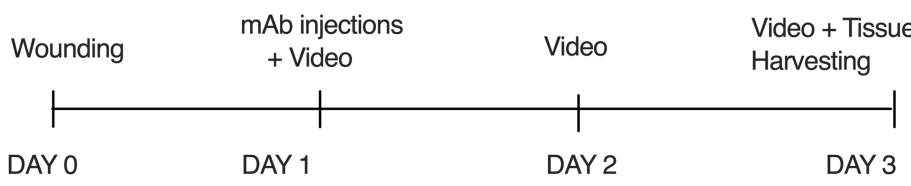

D

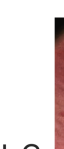

$\lg G$

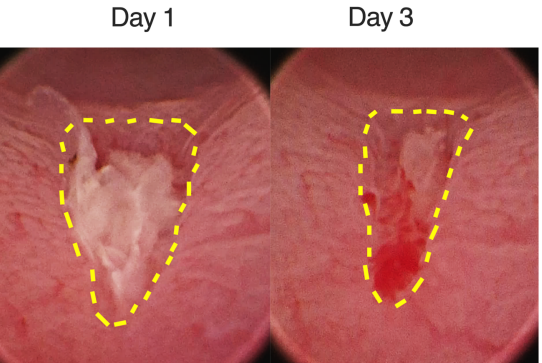

GM35

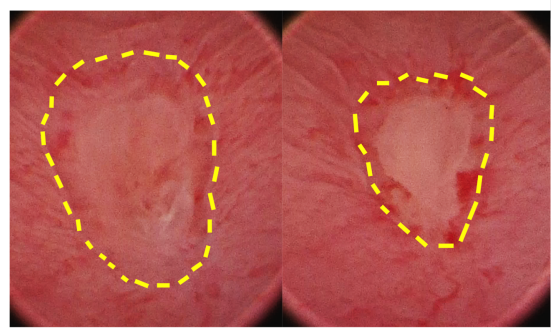

GM35

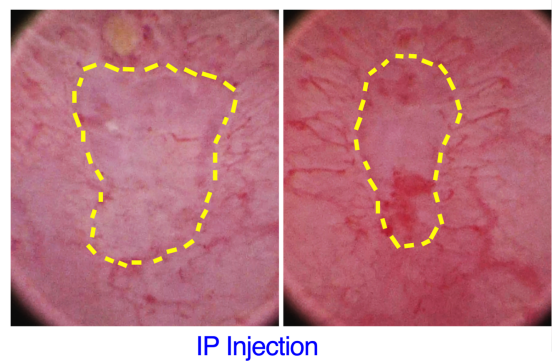

$\lg G$

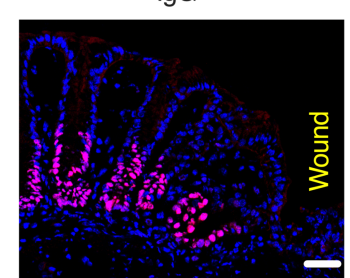

GM35

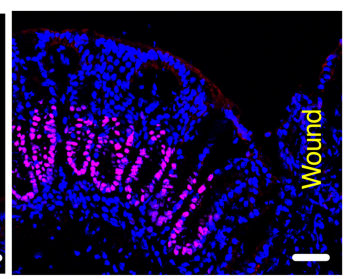

$\star * *$

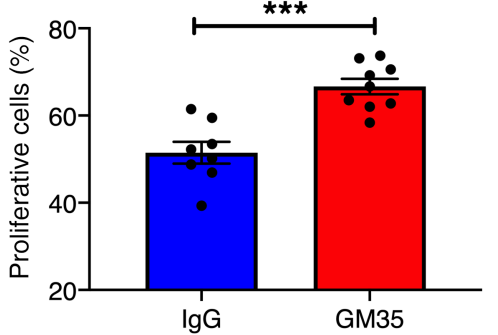

Figure 3. GM35 improves healing of biopsy-induced colonic wounds in vivo. (A) Schematic overview of experimental design timeline. Animals were wounded on day 0 and treated with $\mathrm{GM} 35$ or a control lgG on day 1 via i.p. injection ( $250 \mu \mathrm{g} / \mathrm{animal}$ ) or microinjection directly into the wound bed (10 $\mu \mathrm{g} /$ wound). (B) Percentage of wound healing in animals injected with IgG (blue) or GM35 (red) directly into wound beds. Data are shown as means \pm SEM; $n=3$ independent experiments. ${ }^{* *} P<0.01,{ }^{* * *} P<0.0001$. Mann-Whitney $U$ test for day 2 and unpaired $t$ test for day 3 . (C) Percentage of wound healing in animals i.p. injected with $250 \mu \mathrm{g}$ control lgG (blue) or GM35 (red). Data are shown as means \pm SEM ( $n=3$ independent experiments) and were analyzed by 1-way ANOVA followed by Tukey's post hoc testing. ${ }^{* * *} P<0.0001$. (D) Representative images of wounds at day 1 and day 3 after i.p. injection with lgG control Ab or GM35. (E) Representative immunofluorescence images of colonic wounds stained with anti-CD44v6 mAb (red) and GM35 (green). Scale bar: $10 \mu \mathrm{m}$. Magnification of area adjacent to wound (yellow square) demonstrates expression and colocalization of CD44v6 (red) and sialyl glycan epitopes (GM35, green). (F) Representative immunofluorescence images with proliferation marker Ki67 (pink) in wound-adjacent epithelial crypts after 24 hours of treatment with IgG or GM35. Nuclei are stained in blue. Graph shows quantification of Ki67+ cells in wound-adjacent crypts after treatment with IgG or GM35. Scale bar: $5 \mu \mathrm{m}$. Data are shown as mean \pm SEM ( $n=3$ experiments) and were analyzed by 1-way ANOVA followed by Tukey's post hoc testing. ${ }^{* *} P<0.001$. 

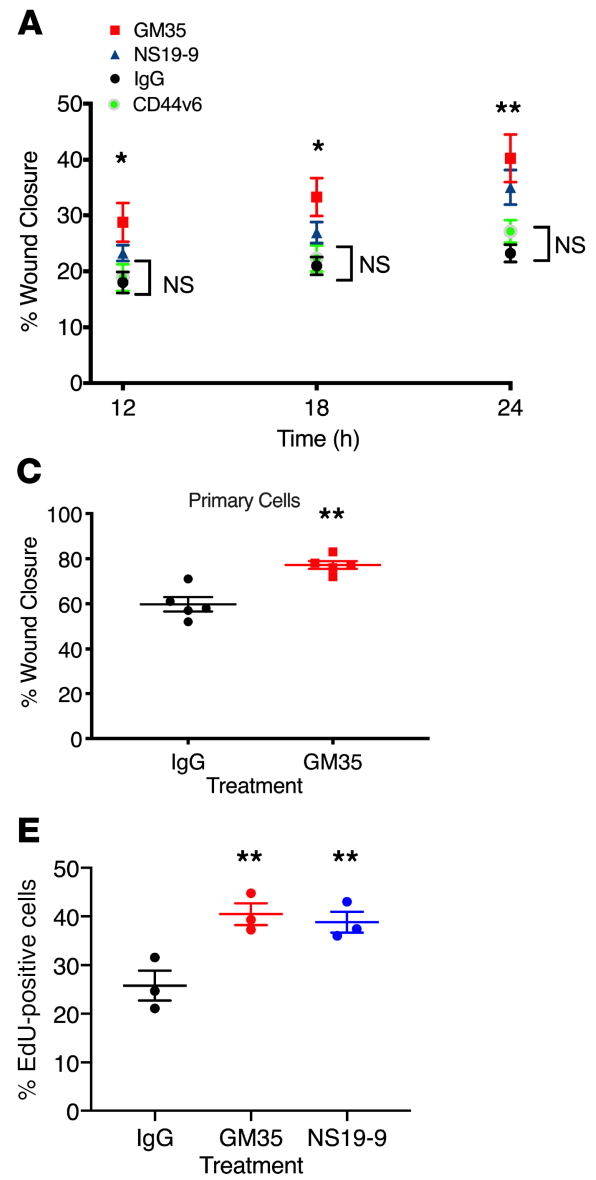

B
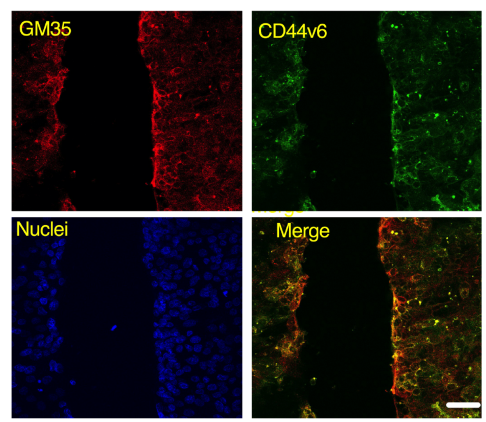

D

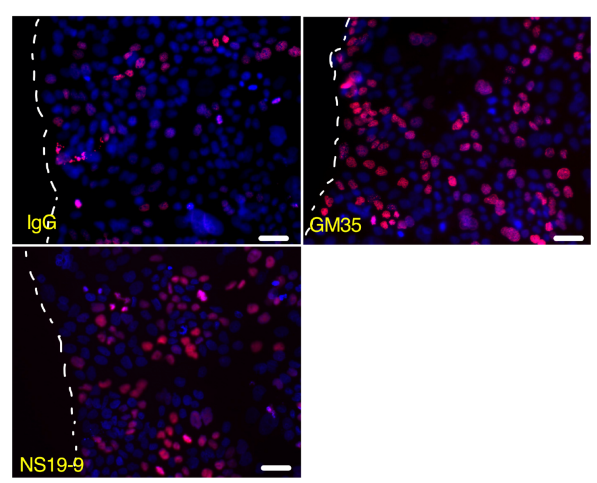

Figure 4. Targeting of sialyl Lewis glycans on CD44v6 with GM35 increases migration and proliferation of human epithelial cells. (A) Percentage of intestinal epithelial wound closure was calculated by measuring wound widths at 12 , 18 , and 24 hours after wounding. IECs were incubated with $10 \mu \mathrm{g} / \mathrm{mL} \mathrm{GM35,} \mathrm{NS19-9,} \mathrm{anti-CD44v6} \mathrm{mAb,} \mathrm{or} \mathrm{IgG} \mathrm{matched}$ control antibody. Data are shown as mean \pm SEM and were analyzed by 1-way ANOVA followed by Tukey's post hoc testing ( $n=4$ experiments, 6 wounds per treatment), ${ }^{*} P<0.05,{ }^{*} P<0.01$. (B) Immunofluorescence staining of scratch-wounded T84 IECs with mAb GM35 in green and anti-CD44v6 mAb in red. Scale bar: $20 \mu \mathrm{m}$. (C) Wound closure after 24 hours was measured in colonoid-derived primary human epithelial cells after treatment with $10 \mu \mathrm{g} / \mathrm{mL}$ GM35 or an IgG matched control mAb. Data are shown as mean \pm SEM analyzed by 1-way ANOVA followed by Tukey's post hoc testing (black and red circles represent averages of $n=5$ experiments, 6 wounds per group per experiment), ${ }^{* *} P<$ 0.01. (D) Scratch-wounded intestinal epithelial monolayers were treated with $10 \mu \mathrm{g} / \mathrm{mL}$ GM35, NS19-9, or control lgG before EdU incorporation was measured 18 hours after injury. Scale bar: $5 \mu \mathrm{m}$. (E) Quantification of proliferation/EdU incorporation. Data are shown as mean \pm SEM analyzed by 1-way ANOVA followed by Tukey's post hoc testing (circles represent averages of $n=3$ experiments, 5 wounds per treatment); ${ }^{* *} P<0.01$.

and E). Consistent with in vivo results, treatment of human epithelial cells with GM35 resulted in a significant increase in the percentage of proliferating cells near wound edges compared with IgG-treated controls $\left(38 \% \pm 6.9 \%\right.$ vs. $\left.27 \% \pm 5.6 \%,{ }^{* *} P<0.01\right)$. A similar increase in proliferation was observed after treatment with another sLe $e^{a}$ binding mAb NS19-9 (39\% $\pm 7.8 \%$ vs. $27 \% \pm 5.6 \%$, $\left.{ }^{* *} P<0.01\right)$. These findings demonstrated that targeting of sialylated Lewis glycans upregulated on wounded human epithelium resulted in increased rates of proliferation and migration, thereby facilitating mucosal repair

Targeting of sialyl Lewis glycans activates intracellular signaling in human IECS. CD44v6 is a known regulator of intracellular signaling events important for proliferation and migration of human epithelial cells (13). Therefore, we assessed the effect of targeting sialyl Lewis glycans on human epithelial intracellular signaling after injury. As shown in Figure 5A, there was increased phosphorylation of Src at Tyr416 18 hours after wounding of human epithelial cells treated with GM35 compared with an IgG control. Similarly, there was increased activation of FAK (Tyr925) and Akt (Thr308 and Ser473) in IECs after ligation of sialyl Lewis glycans on CD44v6 by GM35. By contrast, no activation of p38 MAPK was observed downstream of sialyl Lewis glycan engagement, highlighting the specificity of signaling events mediated by 
A

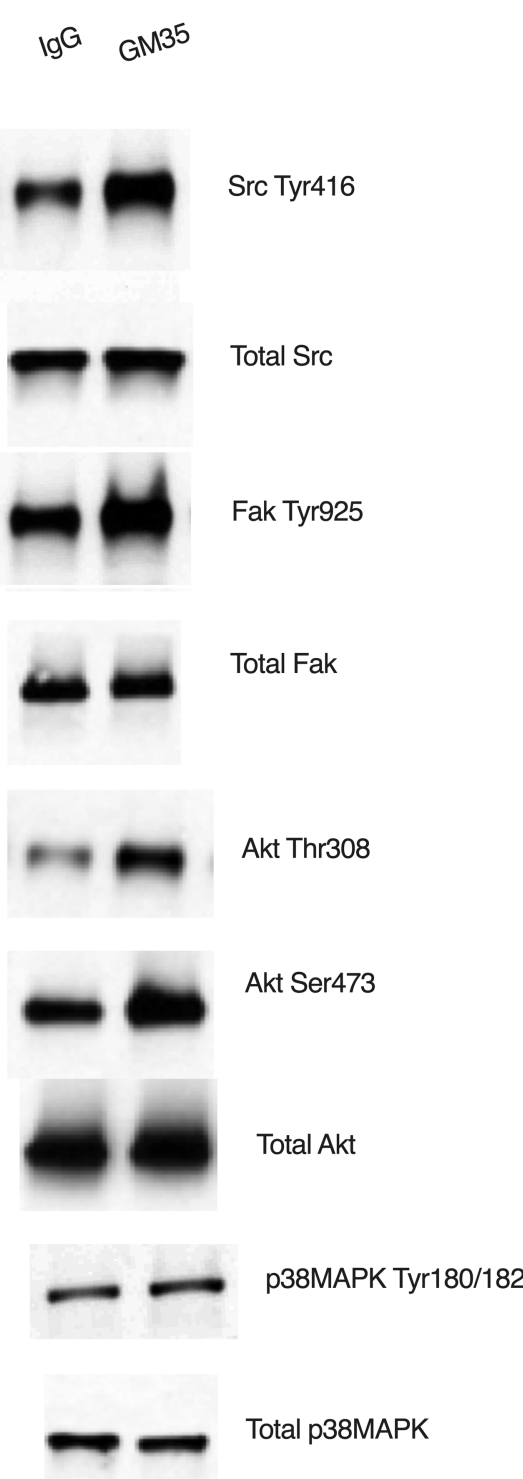

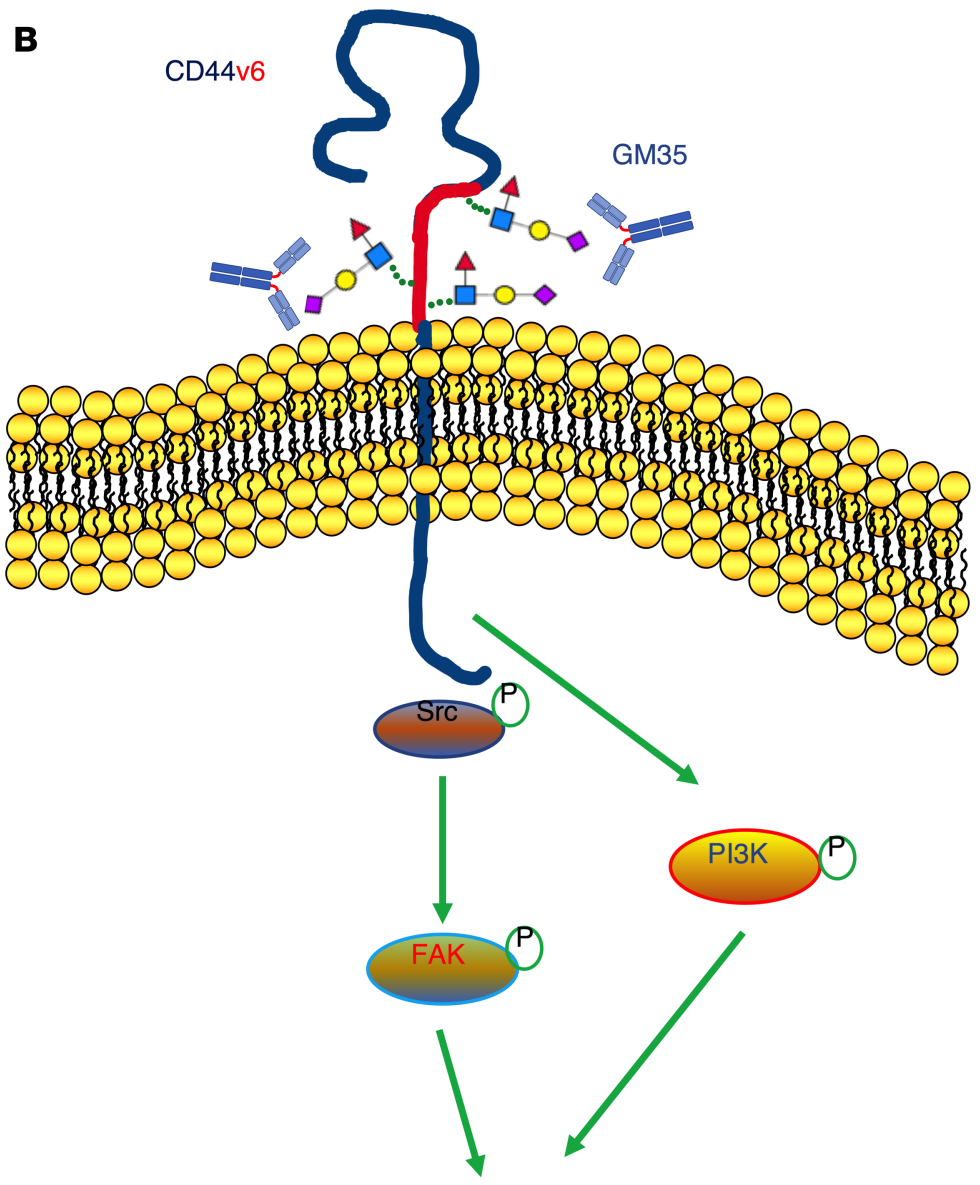

Akt

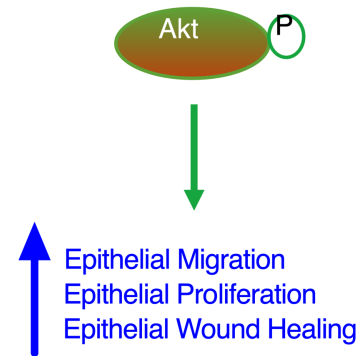

Figure 5. Ligation of sialyI Lewis glycans by GM35 activates proliferation and migration signaling pathways in human IECs. (A) Representative immunoblots from IEC monolayers 18 hours after grid wounding and treatment with $10 \mu \mathrm{g} / \mathrm{mL} \mathrm{GM35}$ or an IgG matched control mAb. Data are representative of $n=$ 3 independent experiments. (B) Proposed model showing GM35 engagement of sialyl Lewis glycans on epithelial CD44v6, resulting in increased activation of Src, FAK, and Akt and leading to increased epithelial proliferation and migration and enhanced wound closure.

GM35. Collectively, these findings support a model shown in Figure 5B, whereby GM35 enhances wound closure by binding to sialyl Lewis glycans on intestinal epithelial CD44v6 to activate intracellular signaling pathways implicated in the regulation of epithelial proliferation and migration.

\section{Discussion}

Delayed intestinal epithelial wound repair is a pathological hallmark of ulcerative colitis and Crohn disease. Chronic intestinal inflammation is also associated with epithelium-specific alterations in glycosylation (7). Using mAb GM35, which is specific for O-glycan-linked sialylated Lewis glycans on epithelial CD44v6 $(6,7)$, we showed robust epithelial expression of immature sialylated glycans in inflamed colonic mucosa from individuals with ulcerative colitis and Crohn disease. This is in contrast to the lack of sLe expression detected in normal, noninflamed colonic mucosa and is supported by previous studies demonstrating overexpression of immature glycans in individuals with active $\operatorname{IBD}(6,7,14)$. In keeping with these data, our previous studies have also demonstrated specific upregulation of epithelial CD $44 \mathrm{v} 6 / \mathrm{sLe}^{\mathrm{a}}$ mediated by 
the proinflammatory cytokine IFN- $\gamma(6)$. Furthermore, previous studies have demonstrated increased sLe $\mathrm{s}^{\mathrm{a}}$ expression in inflamed pancreatic epithelial cells $(15,16)$, suggesting the importance of sLe $\mathrm{e}^{\mathrm{a}}$ as a marker of inflammation in multiple epithelium-lined organs.

Previous work has shown that in addition to binding to the human glycan sLe ${ }^{a}$, GM35 also recognizes a second glycan, the nonfucosylated, biosynthetic precursor of sLe $e^{a}$ termed sLe $e^{c}$, which is expressed in both humans and mice (17-19). We observed increased expression of $\mathrm{sLe}^{\mathrm{c}}$ and $\mathrm{CD} 44 \mathrm{v} 6$ in inflamed murine colonic mucosa, demonstrating that immature or less structurally complex sialylated glycans are indeed markers of epithelial inflammation in both humans and mice. In addition, as has been reported for inflamed human intestinal epithelium, colocalization of murine sialyl Lewis glycans and the epithelium-specific CD44 variant CD44v6 was observed, suggesting protein-specific decoration with these neoglycans in inflamed intestinal mucosa in vivo. Similar colocalization of $s L e^{\mathrm{a}}$ and CD44v6 has been reported in inflamed cervical cells and IECs $(7,20)$. The importance of CD44v6 as a marker of epithelial stress is further highlighted by studies showing that $\mathrm{CD} 44 \mathrm{v} 6$ expression is restricted to inflamed or squamous epithelia $(21,22)$, and that epithelial CD44v6 expression strongly associates with disease activity in ulcerative colitis $(23,24)$.

Importantly, in addition to its use as a marker of inflamed intestinal epithelium, here we showed that systemic treatment with mAb GM35 reduced ulceration associated with DSS-induced colitis in mice. This is consistent with previous studies showing protection of the epithelial barrier in vivo downstream of sialyl Lewis glycan targeting (7). In support of the importance of glycan-mediated signaling events during colitis, others have reported that plant-derived polysaccharides ameliorate colitis in vivo by suppressing NF- $\mathrm{kB}$ signaling and NLRP3 inflammasome activation $(25,26)$. The relevance of glycans as targets to better understand and control mechanisms of intestinal inflammation is further highlighted by studies demonstrating increased levels of highly sialylated glycans in the plasma of individuals with $\operatorname{IBD}(27,28)$. Furthermore, mice with impaired fucosyltransferase gene expression or deficiencies in core-1-derived O-glycans develop an IBD-like spontaneous colitis (29). Chronic inflammation in the gastrointestinal tract is also associated with microbiota-induced changes in host glycosylation that can result in enhanced entry of bacteria and dietary lectins into host tissues (30). Taken together, these studies demonstrate that glycans play a key role in processes fundamental to IBD pathogenesis and that sialylated O-glycans on CD44v6 represent inflammation-induced druggable targets to ameliorate intestinal inflammation.

In addition to protective effects on disease course during colitis, we demonstrated increased epithelial proliferation and migration and improved intestinal epithelial wound closure downstream of targeting sialylated Lewis glycans on CD44v6 in vivo and in vitro. Critically, no effects on epithelial migration were observed downstream of targeting CD44v6 with anti-peptide mAbs, highlighting the importance of CD44v6-expressed sialylated glycans in mediating prorepair effects in the intestinal mucosa. We observed increased expression and colocalization of sialyl Lewis glycans/CD44v6 specifically in epithelial crypts directly adjacent to biopsy-induced wounds in vivo. Consistent with these findings, other studies have demonstrated that after mechanical damage of bronchial epithelial cells, CD44v6 expression increased in areas close to the wound edge (30). A similar localized increase in CD44v6 expression has been reported in damaged areas of lung tissue in asthmatic individuals (31). Injury-induced expression of CD44v6 has also been reported in liver stellate cells, and after activation, these cells engage hyaluronic acid in a CD44v6-dependent manner to enhance cellular momentum (32). In addition to increasing epithelial migration, expression of CD44v6 also correlates with higher proliferative activity in human bladder and pancreatic epithelial cells $(33,34)$.

Here we report changes in posttranslational glycosylation, in addition to increased protein expression of CD44v6 after colonic epithelial injury. The importance of glycosylation to wound repair is highlighted by previous studies reporting glycan-mediated increases in epithelial proliferation and wound repair. Specifically, plant root polysaccharides have been shown to promote proliferation, migration, and cell cycle progression in murine and human models of skin wounding (35). Similarly, $\beta$-glucan polysaccharides promote migration of dermal fibroblasts, resulting in improved healing of murine skin wounds (36). Previous studies have also highlighted a role for sialylated glycans in enhancing epithelial mobility. As such, treatment of mammary carcinoma cells with sialidase to remove terminal sialic acids resulted in decreased epithelial cell matrix adhesion interactions and reduced cell motility (37). Other studies have shown that cell surface sialyl O-glycans on human colon cancer cells regulate intercellular adhesion events and play an important role in tumor migration. Taken together, these studies support the importance of sialylated Lewis glycan/CD44v6-mediated cellular adhesion events in promoting epithelial migration and proliferation and increasing mucosal wound healing in the intestine. 
It is well documented that CD44v6 ligation activates multiple downstream intracellular signaling molecules to modulate epithelial cell function $(38,39)$. Furthermore, several kinases, including Akt, Src, and FAK, have been identified as being downstream signaling mediators of CD44v6 (40-44). In support of these previous reports, we showed that intestinal epithelial activation of Src, FAK, and Akt downstream of GM35 mediated ligation of sLe $\mathrm{e}^{\mathrm{a}}$ on $\mathrm{CD} 44 \mathrm{v} 6$. Our observations are consistent with previous work reporting that increased CD44v6 expression results in Akt activation in oral epithelial cells (43) and another study of pancreatic epithelial cells that reported downstream signaling through FAK and PI3K after engagement of CD44v6 (40).

The data presented here demonstrated inflammation-induced increases in expression of immature sialylated Lewis glycans on intestinal epithelium-expressed CD44v6, and that selective antibody-mediated targeting of these glycans on colonic epithelial cells activated intracellular signaling pathways known to promote epithelial proliferation, migration, and wound closure $(40,43)$. Although commercial antibodies for $\mathrm{sLe}^{\mathrm{a}}$ are available, they have a much lower affinity than mAb GM35 (7) and, importantly, do not recognize the murine glycan sLe $\mathrm{s}^{\mathrm{c}}$ (http://www.functionalglycomics.org/glycomics/HServlet?operation=view\&side$\mathrm{Menu}=$ no\&psId=primscreen_3256). Taken together, these findings suggest that targeting sialylated Lewis glycans on epithelial CD44v6 may represent a promising new therapeutic strategy for improved recovery of epithelial barrier function and restitution of intestinal homeostasis after inflammation or injury.

\section{Methods}

Animals. Experimental studies were performed in C57BL/6 WT mice bred at University of Michigan animal resource facilities or purchased from The Jackson Laboratory. Animals (10-14 weeks, both sexes) were maintained under specific pathogen-free conditions with a 12-hour light/12-hour dark cycle and access to food and water ad libitum.

Epithelial cell lines and human colonoids. T84 IECs were grown under standard conditions as previously described (45). Human colonoids were generated from biopsies of normal human colonic mucosa as previously described $(46,47)$. All human colon sample collection was performed in accordance with the University of Michigan IRB regulations. Isolated colonoids were resuspended in Matrigel and cultured in growth medium (50\% L-WRN conditioned medium: 50\% advanced DMEM/F-12, 10\% FBS, 2 mM GlutaMax, $10 \mathrm{mM}$ HEPES, N-2 media supplement, B-27 supplement, $1 \mathrm{mM}$ N-acetyl-L-cysteine, $50 \mathrm{ng} / \mathrm{mL}$ human EGF, 100 $\mathrm{U} / \mathrm{mL}$ penicillin, $0.1 \mathrm{mg} / \mathrm{mL}$ streptomycin, $500 \mathrm{nM}$ A83-01, $10 \mu \mathrm{m}$ SB202190, $10 \mathrm{mM}$ nicotinamide, $10 \mathrm{nM}$ gastrin). To generate 2D monolayers, colonoids grown as described above were spun out of Matrigel and dissociated into a single-cell suspension according to published protocols (48). After 1 day in complete growth medium (as described above), epithelial cells were switched to differentiation media (growth medium minus Wnt3a, R-spondin, nicotinamide, and SB202190 and with a 50\% reduction in noggin) for 4-5 days of differentiation into confluent monolayers of colonoid epithelium

Antibodies. mAb GM35 was isolated as described previously (6, 7). Monoclonal anti-human CD44v6 $\mathrm{mAb}$ was purchased from R\&D Systems, Bio-Techne (catalog BBA1), and anti-mouse CD44v6 mAb was ordered from eBioscience (catalog BMS145). Monoclonal anti-sLe ${ }^{a}$ mAb NS19-9 was purchased from Origene (catalog 190083). Anti-Muc-2 mAb was purchased from Abcam (catalog ab90007).

DSS-induced colitis and histological scoring. For DSS-induced colitis experiments, mice were i.p. injected with $250 \mu \mathrm{g}$ of mAb GM35 or isotype control mAb (BD Biosciences, 554721) 1 day before and 2 days after administration of DSS. Next, $2.5 \%$ DSS was added to drinking water and disease activity was calculated daily for 8 days by measuring body weight, stool consistency, and the presence of occult blood (49). For H\&E staining, 6- $\mu \mathrm{m}$ sections were used. Two independent investigators analyzed histology for total length and areas of inflammation and ulceration by Aperio ImageScope 12 (Leica Biosystems). Subsequently, percentages of inflamed and ulcerated areas were calculated and a final score generated using a validated scoring system (50).

For DSS-induced colitis experiments with no mAb pretreatment, mice were placed on $3 \%$ DSS for 5 days, followed by 4 days of water. Mice were i.p. injected with $250 \mu \mathrm{g}$ of mAb GM35 or isotype control mAb (BD Biosciences, 554721), or PBS alone on days 1, 3, 6, and 8 after administration of DSS. Disease activity and mucosal injury scores were calculated as described above.

PMN TEpM into the colon in vivo. Colon loop experiments were performed with C56BL/6 WT mice (8-12 weeks, both sexes) which were maintained under standard conditions with 12-hour light/12-hour dark cycles and ad libitum access to food and water. To quantify PMN TEpM into the proximal colon in vivo, a recently published protocol was applied (12). Briefly, mice were pretreated with proinflammatory 
cytokines before a 2-cm loop of vascularized proximal colon was injected with 1 nM LTB4 with GM35 or an IgG control $\mathrm{mAb}$ before migration of PMNs into the intestinal lumen was quantified by flow cytometry.

In vivo wounding of colonic mucosa. Biopsy wounding of colonic mucosa in mice was performed as previously described (51). Briefly, mice were anesthetized by an i.p. injection of a ketamine (100 mg/kg)/ xylazine $(10 \mathrm{mg} / \mathrm{kg})$ solution. Biopsy-induced injuries of the colonic mucosa were made along the mesenteric artery using a high-resolution, miniaturized colonoscope system equipped with a biopsy forceps (Colorview Veterinary Endoscope, Karl Storz), and 6-10 lesions were generated per animal. Endoscopic procedures were viewed on days 1,2 , and 3 after injury with high-resolution images $(1024 \times 768$ pixels) on a flat-panel monitor. $250 \mu \mathrm{g}$ mAb GM35 or isotype control antibodies (BD Biosciences, catalog 554721) were i.p. injected 1 day after biopsy wounding. For wound bed injections, $10 \mu \mathrm{g}$ of mAb GM35 or isotype control $\mathrm{mAb}$ was directly injected into wound beds using a modified 30 -gauge needle (30 $\mathrm{G} \times$ $1 / 2$; Becton Dickinson). To analyze proliferation, antibodies were i.p. injected immediately after wounding. Subsequently, mucosal wounds and intact tissues were harvested for immunofluorescence labeling, immunoblotting, and flow cytometry analyses.

Lamina propria isolation and immune cell staining. To isolate immune cells from wounds, 5 wounds per animal were removed by punch biopsy ( $3 \mathrm{~mm}$, Disposable Biopsy Punch, Integra Miltex, catalog 33-32) and pooled together. Tissues were minced and digested with Liberase TM (2.5 mg/mL; Roche Diagnostics) and DNase I (MilliporeSigma) under mechanical agitation. After filtering, cell suspensions were transferred to a 96-well plate and stained for indicated immune cell populations. For quantification, a NovoCyte Flow Cytometer (ACEA Bioscience) was used and data analyses were performed using FlowJo version 10 software. After exclusion of cell doublets by gating FSC area (FSC-A) versus FSC height (FSH-H), cells were initially gated for $\mathrm{CD} 45^{+}$events and $\mathrm{CD} 11 \mathrm{~b}$ expression to only include myeloid cells. Following that, cells were gated for Ly6G-1A8 (PMNs) and SiglecF (eosinophils). Ly6G/SiglecF-double negative cells were further gated for F4/80 (macrophages) and Ly6C (monocytes).

Immunofluorescence and immunohistochemistry. For immunofluorescence labeling with mAb GM35 and anti-CD44v6 mAbs (eBioscience, catalog BMS145), tissues were formalin fixed and embedded in paraffin. Sections were cut at 5 - $\mu \mathrm{m}$ thickness followed by deparaffinization, rehydration, and antigen retrieval with Antigen Unmasking Solution (1:100, Vector Laboratories, catalog H3301). After blocking in 1\% BSA (MilliporeSigma, catalog A3059), sections were incubated with primary antibody at $4^{\circ} \mathrm{C}$ overnight. The next day, slides were incubated with secondary antibody (1:500) for 45 minutes and Hoechst stain (1:1000, Invitrogen, Thermo Fisher Scientific, catalog H3570) to visualize nuclei before being mounted with ProLong Gold antifade reagent (Invitrogen, Thermo Fisher Scientific, catalog P36934). For Ki67 staining (Abcam, ab15580), OCT-embedded sections $(6 \mu \mathrm{m})$ were fixed in $4 \%$ paraformaldehyde and permeabilized using $0.5 \%$ Triton X-100 (MilliporeSigma). After blocking, sections were incubated with primary antibody $\mathrm{Ki67}(1: 100)$ at $4^{\circ} \mathrm{C}$ overnight. Subsequently, slides were incubated with a secondary antibody (1:500) and Hoechst stain (1:1000) before mounting with ProLong Gold antifade reagent. Immunofluorescence images were taken by confocal microscopy (Leica SP5 Inverted 2-Photon FLIM Confocal). ImageJ (NIH) was used for image processing. For human tissue staining, paraffin-embedded sections $(6 \mu \mathrm{m})$ slides of discarded resections from patients with ulcerative colitis, Crohn disease, or negative colorectal screens were obtained with approval from the University of Michigan IRB. Tissue sections were incubated with GM35 at $10 \mu \mathrm{g} / \mathrm{mL}$ followed by H\&E staining. Images were captured using an IX73 microscope system.

Human IEC monolayer wounding. Migration of T84 IECs and human colonoid-derived monolayers was assessed using a scratch wound assay as previously described (52). Then, $10 \mu \mathrm{g} / \mathrm{mL}$ GM35, NS19-9, anti-CD44v6 mAb, or IgG control mAbs were added directly after wounding and epithelial migration and wound healing were measured after 24 hours. For immunoblot analysis, confluent T84 cell monolayers grown in 6-well tissue culture plates were grid wounded to enrich for migrating and spreading cells as previously described (51).

Epithelial cell proliferation assays. Confluent T84 IEC monolayers were scratch wounded with a sterile pipette tip (as described above) before 18 hours of treatment with $10 \mu \mathrm{g} / \mathrm{mL}$ GM35, NS19-9, or IgG control mAbs. One hour before fixing of cells, 5-ethynyl-2'-deoxyuridine (EdU) was added to the media at a concentration of $10 \mu \mathrm{m}$. Proliferating cells were detected with the Click-iT EdU Alexa Fluor 594 Imaging kit (Life Sciences, Thermo Fisher Scientific; catalog C10639) according to the manufacturer's instructions and captured using a Leica SP5 confocal microscope (Leica Microsystems). 
Cell lysis and immunoblotting. For cell lysis, IECs were harvested in RIPA buffer $(0.5 \%$ Triton X-100, $0.5 \%$ NP-40, 0.5\% deoxycholic acid, $0.1 \%$ SDS, $150 \mathrm{mM} \mathrm{NaCl}, 1 \mathrm{mM}$ EGTA [pH 8.0], $0.2 \mathrm{mM}$ sodium orthovanadate, $20 \mathrm{mM}$ Tris [pH 7.4]) supplemented with protease and phosphatase inhibitors. Immunoblotting was performed as described previously $(7,53)$.

Statistics. Statistical analyses were performed using Prism software (GraphPad Software Inc.). A 2-tailed Student's $t$ test was used in case of parametric parameters. For nonparametric data, differences were evaluated by Mann-Whitney $U$ test or 1-way ANOVA followed by Tukey's post hoc test. A $P$ value of less than 0.05 was considered statistically significant. Data are presented as means \pm SEM. All results show data from at least 3 independent experiments.

Study approval. All experimental procedures involving animals were conducted in accordance with NIH guidelines and protocols approved by the University Committee on Use and Care of Animals at the University of Michigan. All human colon sample collection was performed with written informed consent in accordance with the University of Michigan Institutional Review Board regulations.

\section{Author contributions}

$\mathrm{MK}, \mathrm{MQ}, \mathrm{VA}, \mathrm{KB}$, and JCB designed the study and performed data collection and data analysis/interpretation. JCB wrote the manuscript. RDC provided assistance with interpretation of glycan binding data. AN and CAP provided assistance in writing the manuscript.

\section{Acknowledgments}

The authors would like to thank the National Center for Functional Glycomics (NCFG) at Beth Israel Deaconess Medical Center, Harvard Medical School (supporting grant P41 GM103694). The authors would like to thank the Translational Tissue Modeling Laboratory (TTML) at University of Michigan for providing human colonoids. This work was supported by Crohn's and Colitis Foundation (544596) funding to JCB; German Research Foundation/DFG (KE2402/2-1) funding to MK; NIH DK59888 and DK55679 to AN; and NIH DK072564, DK079392, and DK061379 to CAP.

Address correspondence to: Jennifer Brazil, 109 Zina Pitcher Place, BSRB Room 4620, Ann Arbor, Michigan 48104, USA. Phone: 734.936.1856; Email: brazilj@med.umich.edu.

1. Quirós M, Nusrat A. Contribution of wound-associated cells and mediators in orchestrating gastrointestinal mucosal wound repair. Annu Rev Physiol. 2019;81:189-209.

2. Dignass AU, Podolsky DK. Cytokine modulation of intestinal epithelial cell restitution: central role of transforming growth factor beta. Gastroenterology. 1993;105(5):1323-1332.

3. Sturm A, Dignass AU. Epithelial restitution and wound healing in inflammatory bowel disease. World J Gastroenterol. 2008;14(3):348-353.

4. Senbanjo LT, Chellaiah MA. CD44: A multifunctional cell surface adhesion receptor is a regulator of progression and metastasis of cancer cells. Front Cell Dev Biol. 2017;5:18.

5. Wang Z, Zhao K, Hackert T, Zöller M. CD44/CD44v6 a reliable companion in cancer-initiating cell maintenance and tumor progression. Front Cell Dev Biol. 2018;6:97.

6. Brazil JC, Lee WY, Kolegraff KN, Nusrat A, Parkos CA, Louis NA. Neutrophil migration across intestinal epithelium: evidence for a role of CD44 in regulating detachment of migrating cells from the luminal surface. J Immunol. 2010;185(11):7026-7036.

7. Brazil JC, et al. $\alpha 3 / 4$ Fucosyltransferase 3-dependent synthesis of sialyl Lewis A on CD44 variant containing exon 6 mediates polymorphonuclear leukocyte detachment from intestinal epithelium during transepithelial migration. J Immunol. 2013;191(9):4804-4817.

8. Paclik D, Lohse K, Wiedenmann B, Dignass AU, Sturm A. Galectin-2 and -4, but not galectin-1, promote intestinal epithelial wound healing in vitro through a TGF-beta-independent mechanism. Inflamm Bowel Dis. 2008;14(10):1366-1372.

9. Vagin O, Tokhtaeva E, Yakubov I, Shevchenko E, Sachs G. Inverse correlation between the extent of N-glycan branching and intercellular adhesion in epithelia. Contribution of the Na,K-ATPase beta1 subunit. J Biol Chem. 2008;283(4):2192-2202.

10. Bettess MD, et al. c-Myc is required for the formation of intestinal crypts but dispensable for homeostasis of the adult intestinal epithelium. Mol Cell Biol. 2005;25(17):7868-7878.

11. Kiesler P, Fuss IJ, Strober W. Experimental models of inflammatory bowel diseases. Cell Mol Gastroenterol Hepatol. 2015;1(2):154-170.

12. Flemming S, Luissint AC, Nusrat A, Parkos CA. Analysis of leukocyte transepithelial migration using an in vivo murine colonic loop model. JCI Insight. 2018;3(20):99722.

13. Orian-Rousseau V, Sleeman J. CD44 is a multidomain signaling platform that integrates extracellular matrix cues with growth factor and cytokine signals. Adv Cancer Res. 2014;123:231-254.

14. Larsson JM, et al. Altered O-glycosylation profile of MUC2 mucin occurs in active ulcerative colitis and is associated with increased inflammation. Inflamm Bowel Dis. 2011;17(11):2299-2307. 
15. Itai S, et al. Significance of 2-3 and 2-6 sialylation of Lewis A antigen in pancreas cancer. Cancer. 1988;61(4):775-787.

16. Itai S, et al. Tissue distribution of 2-3 and 2-6 sialyl Lewis A antigens and significance of the ratio of two antigens for the differential diagnosis of malignant and benign disorders of the digestive tract. Cancer. 1991;67(6):1576-1587.

17. Narimatsu H, et al. Lewis and secretor gene dosages affect CA19-9 and DU-PAN-2 serum levels in normal individuals and colorectal cancer patients. Cancer Res. 1998;58(3):512-518.

18. Hennet T, Dinter A, Kuhnert P, Mattu TS, Rudd PM, Berger EG. Genomic cloning and expression of three murine UDP-galactose: beta-N-acetylglucosamine beta1,3-galactosyltransferase genes. J Biol Chem. 1998;273(1):58-65.

19. Kim KS, Oh SU, Lee JH, Lee YC. Mutational analysis for enzyme activity of mouse Galbeta1,3GalNAc alpha2,3-sialyltransferase (mST3Gal I). Indian J Biochem Biophys. 2010;47(3):135-140.

20. Salinas-Muñoz L, et al. Estrogen receptor-alpha (ESR1) governs the lower female reproductive tract vulnerability to Candida albicans. Front Immunol. 2018;9:1033.

21. Salmi M, Grön-Virta K, Sointu P, Grenman R, Kalimo H, Jalkanen S. Regulated expression of exon v6 containing isoforms of CD44 in man: downregulation during malignant transformation of tumors of squamocellular origin. $J$ Cell Biol. 1993;122(2):431-442.

22. Terpe HJ, Stark H, Prehm P, Günthert U. CD44 variant isoforms are preferentially expressed in basal epithelial of non-malignant human fetal and adult tissues. Histochemistry. 1994;101(2):79-89.

23. Crocker PR, Feizi T. Carbohydrate recognition systems: functional triads in cell-cell interactions. Curr Opin Struct Biol. 1996;6(5):679-691.

24. Camacho FI, Muñoz C, Sánchez-Verde L, Sáez AI, Alcántara M, Rodríguez R. CD44v6 expression in inflammatory bowel disease is associated with activity detected by endoscopy and pathological features. Histopathology. 1999;35(2):144-149.

25. Cui L, et al. Polysaccharide from Scutellaria baicalensis Georgi ameliorates colitis via suppressing NF- $\mathrm{kB}$ signaling and NLRP3 inflammasome activation. Int J Biol Macromol. 2019;132:393-405.

26. Lv J, et al. Astragalus polysaccharides protect against dextran sulfate sodium-induced colitis by inhibiting NF- $\mathrm{B}$ activation. Int J Biol Macromol. 2017;98:723-729.

27. Clerc F, et al. Plasma N-glycan signatures are associated with features of inflammatory bowel diseases. Gastroenterology. 2018;155(3):829-843.

28. McGovern DP, et al. Fucosyltransferase 2 (FUT2) non-secretor status is associated with Crohn's disease. Hum Mol Genet. 2010;19(17):3468-3476.

29. Fu J, et al. Loss of intestinal core 1-derived O-glycans causes spontaneous colitis in mice. J Clin Invest. 2011;121(4):1657-1666.

30. Goto Y, Uematsu S, Kiyono H. Epithelial glycosylation in gut homeostasis and inflammation. Nat Immunol. 2016;17(11):1244-1251.

31. Lackie PM, Baker JE, Günthert U, Holgate ST. Expression of CD44 isoforms is increased in the airway epithelium of asthmatic subjects. Am J Respir Cell Mol Biol. 1997;16(1):14-22.

32. Kikuchi S, Griffin CT, Wang SS, Bissell DM. Role of CD44 in epithelial wound repair: migration of rat hepatic stellate cells utilizes hyaluronic acid and CD44v6. J Biol Chem. 2005;280(15):15398-15404.

33. Kuncová J, Kostrouch Z, Viale M, Revoltella R, Mandys V. Expression of CD44v6 correlates with cell proliferation and cellular atypia in urothelial carcinoma cell lines 5637 and HT1197. Folia Biol (Praha). 2005;51(1):3-11.

34. Tsuchida A, et al. Significance of CD44s and CD44v6 expression in pancreaticobiliary maljunction. Hepatogastroenterology. 2011;58(112):1877-1881.

35. Zhao B, Zhang X, Han W, Cheng J, Qin Y. Wound healing effect of an Astragalus membranaceus polysaccharide and its mechanism. Mol Med Rep. 2017;15(6):4077-4083.

36. Fusté NP, et al. Barley $\beta$-glucan accelerates wound healing by favoring migration versus proliferation of human dermal fibroblasts. Carbohydr Polym. 2019;210:389-398.

37. Wang XY, Lin SQ, Li JW, Kemmner W, Ding YQ. [Effect of cell surface sialic acid and their linkages on adhesion of mammary carcinoma cells]. Nan Fang Yi Ke Da Xue Xue Bao. 2006;26(6):742-746.

38. Wang Y, Yang X, Xian S, Zhang L, Cheng Y. CD44v6 may influence ovarian cancer cell invasion and migration by regulating the NF-кB pathway. Oncol Lett. 2019;18(1):298-306.

39. Lee JL, Wang MJ, Sudhir PR, Chen JY. CD44 engagement promotes matrix-derived survival through the CD44-SRC-integrin axis in lipid rafts. Mol Cell Biol. 2008;28(18):5710-5723.

40. Jung T, Gross W, Zöller M. CD44v6 coordinates tumor matrix-triggered motility and apoptosis resistance. J Biol Chem. 2011;286(18):15862-15874.

41. Jijiwa M, et al. CD44v6 regulates growth of brain tumor stem cells partially through the AKT-mediated pathway. PLoS One. 2011;6(9):e24217.

42. Chung SY, Huang WC, Chen ZS, Chao TC, Su Y. Elucidation of the mechanism underlying CD44v6-induced transformation of IEC-6 normal intestinal epithelial cells. J Cell Physiol. 2020;235(1):194-209.

43. Kashyap T, et al. Crosstalk between Raf-MEK-ERK and PI3K-Akt-GSK3 $\beta$ signaling networks promotes chemoresistance, invasion/migration and stemness via expression of CD44 variants (v4 and v6) in oral cancer. Oral Oncol. 2018;86:234-243.

44. Cary LA, Klinghoffer RA, Sachsenmaier C, Cooper JA. SRC catalytic but not scaffolding function is needed for integrin-regulated tyrosine phosphorylation, cell migration, and cell spreading. Mol Cell Biol. 2002;22(8):2427-2440.

45. Parkos CA, Delp C, Arnaout MA, Madara JL. Neutrophil migration across a cultured intestinal epithelium. Dependence on a CD11b/CD18-mediated event and enhanced efficiency in physiological direction. J Clin Invest. 1991;88(5):1605-1612.

46. Sato T, Clevers H. Growing self-organizing mini-guts from a single intestinal stem cell: mechanism and applications. Science. 2013;340(6137):1190-1194.

47. Sato T, et al. Single Lgr5 stem cells build crypt-villus structures in vitro without a mesenchymal niche. Nature. 2009;459(7244):262-265.

48. Zou WY, et al. Human intestinal enteroids: new models to study gastrointestinal virus infections. Methods Mol Biol. 2019;1576:229-247

49. Krieglstein CF, et al. Collagen-binding integrin alpha1beta1 regulates intestinal inflammation in experimental colitis. J Clin Invest. 2002;110(12):1773-1782. 
50. Khounlotham M, et al. Compromised intestinal epithelial barrier induces adaptive immune compensation that protects from colitis. Immunity. 2012;37(3):563-573.

51. Quiros M, et al. Macrophage-derived IL-10 mediates mucosal repair by epithelial WISP-1 signaling. J Clin Invest. 2017;127(9):3510-3520.

52. Leoni G, et al. Annexin A1, formyl peptide receptor, and NOX1 orchestrate epithelial repair. J Clin Invest. 2013;123(1):443-454.

53. Misra S, et al. Hyaluronan-CD44 interactions as potential targets for cancer therapy. FEBS J. 2011;278(9):1429-1443. 